

This is an informal report intended for use as a preliminary or working document

\title{
GEND
}

General Public Utilities - Gectric Power Research Institute U.S. Nuclear Regulatory Commission U.S. Department of Energy

Quick Look Report

Entry 4

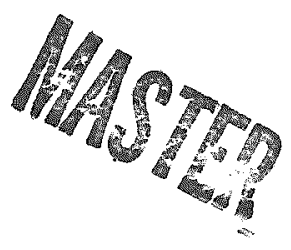

Three Mile Island Unit 2

November 13, 1980

\section{G.E. Eidam}

Prepared for the

U. S. Department of Energy

Three Mile Island Operations Office

Under Contract No. DE-AC07-76ID01570 


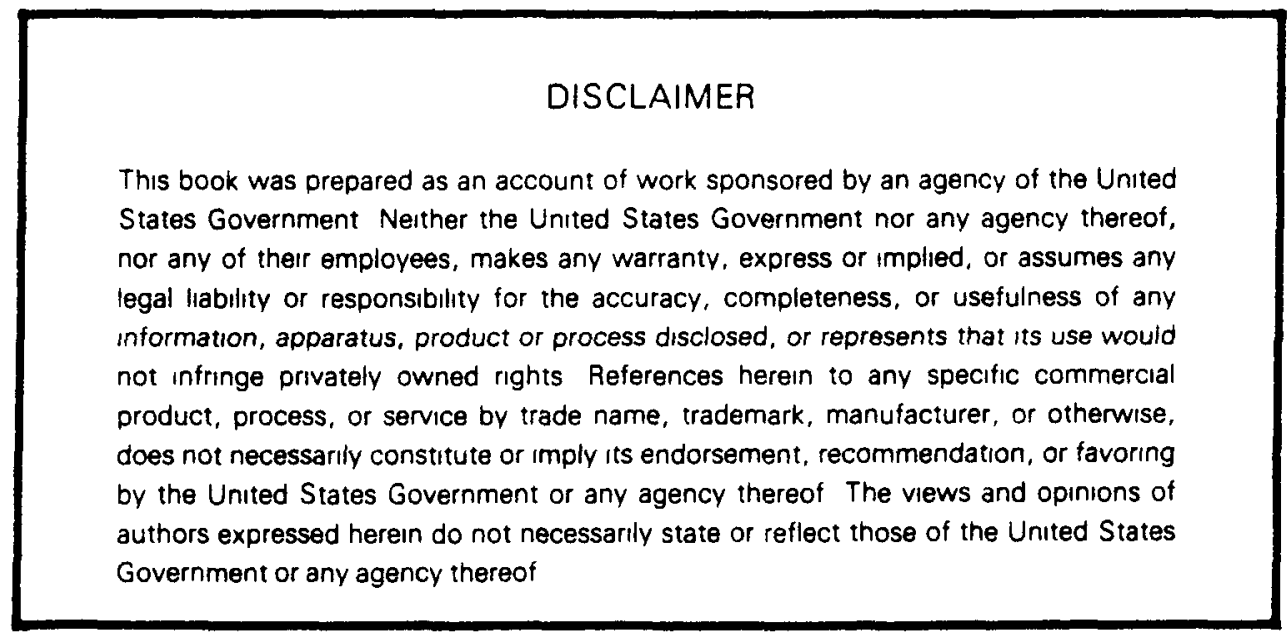




\section{DISCLAIMER}

This report was prepared as an account of work sponsored by an agency of the United States Government. Neither the United States Government nor any agency Thereof, nor any of their employees, makes any warranty, express or implied, or assumes any legal liability or responsibility for the accuracy, completeness, or usefulness of any information, apparatus, product, or process disclosed, or represents that its use would not infringe privately owned rights. Reference herein to any specific commercial product, process, or service by trade name, trademark, manufacturer, or otherwise does not necessarily constitute or imply its endorsement, recommendation, or favoring by the United States Government or any agency thereof. The views and opinions of authors expressed herein do not necessarily state or reflect those of the United States Government or any agency thereof. 


\section{DISCLAIMER}

Portions of this document may be illegible in electronic image products. Images are produced from the best available original document. 
GEND-INF-004

TIO-QLR

\section{QUICK LOOK REPORT ENTRY 4 \\ THREE MILE ISLAND UNIT 2 , NOVEMBER 13, 1980}

G. E. Eidam

Published June 1981
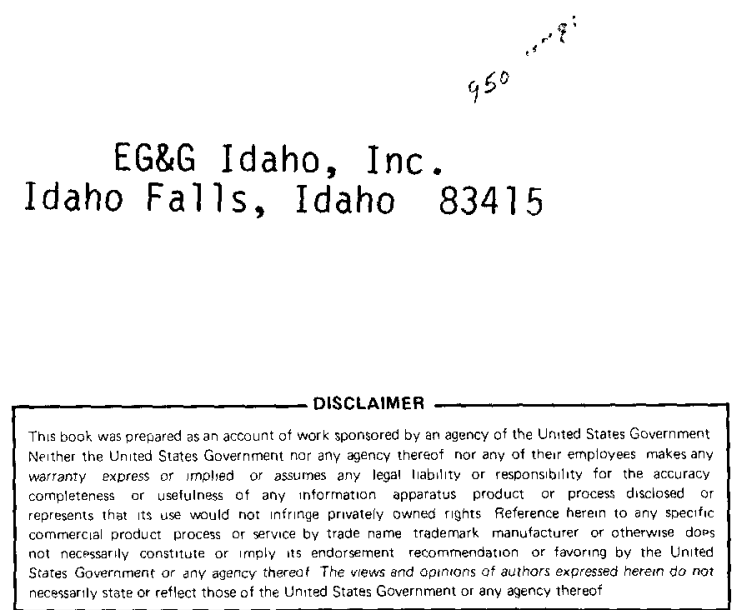

Prepared for the

U.S. Department of Energy

Three Mile Island Operations Office

Under Contract No. DE-AC07-ID01570 


\section{ABSTRACT}

This report summarizes tasks performed during entry 4 at Three Mile Island Unit 2. During the entry into containment, which was made on November 13, 1980, additional beta and gamma surveys were conducted to supplement data acquired on previous entries. A decontamination test was completed on Elevation 305. Power receptables tested on Elevation 305 were deenergized, but receptacles on Elevation 347 were energized.

Still photography was acquired of Elevations 305 and 347 . During the entry, 86 still photographs were taken. Videotaping (color and black and white) was done on Elevations 305 and 347 , but lighting on both elevations was insufficient for high-quality video. 


\section{FOREWORD}

The frequency of entries into containment at Three Mile Island Unit 2 is increasing, as are the complexity and number of tasks undertaken during each entry. For these reasons a brief summary of tasks and analytical resuits organized by entry is needed. This series of entry Quick Look Reports (QLRS) meets the need.

At the time of publication of this report, additional QLRs covering Entries 1 through 7 have been published. A QLR will be published following each subsequent entry. 


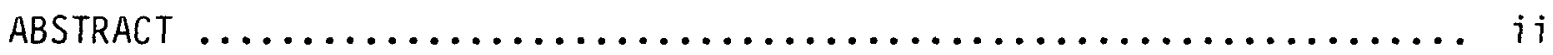

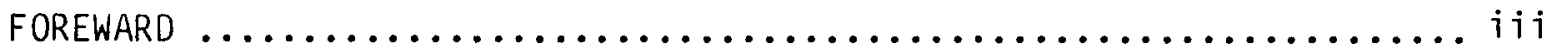

ENTRY OVERVIEW $\ldots \ldots \ldots \ldots \ldots \ldots \ldots \ldots \ldots \ldots \ldots \ldots \ldots \ldots \ldots \ldots \ldots \ldots$

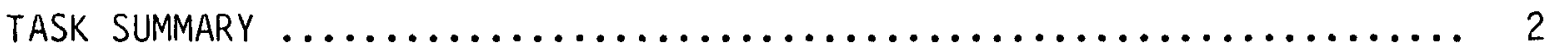

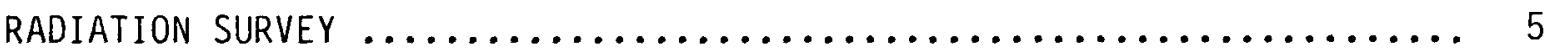

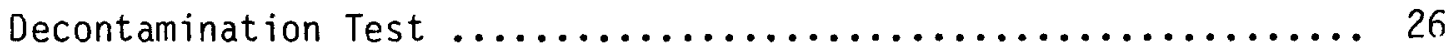

Airborne Activity $\ldots \ldots \ldots \ldots \ldots \ldots \ldots \ldots \ldots \ldots \ldots \ldots \ldots \ldots \ldots$

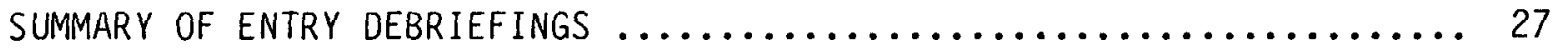

\section{FIGURES}

1. Radiation survey locations on Elevation 305 for Entry 4, November 13, 1980. (Refer to Table 2.) ..................... 16

2. Radiation sur'ey location on Elevation 305 for Entry 4, November 1?, 1980. This is the HPR-211 area survey, located on the enclosed stairwell wall, as indicated in Figure 1.

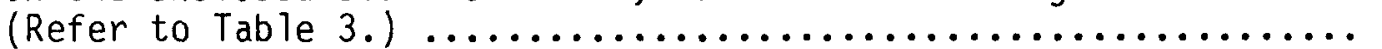

3. Radiation survey locations on Elevation 305 for Entry 4, November 13, 1980. These survey locations are indicated on the plan view of the reactor builcing equipment hatch.

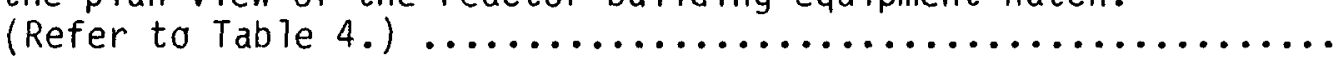

4. Radiation survey locations on Elevation 305 for Entry 4, November 13,1980. These survey points are indicated on the plan view of the reactor building Personnel Airlock (PAL) \#2.

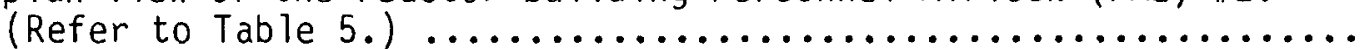

5. Radiation survey location on Elevation 305 (data points 16-28 are at Elevation 367) for Entry 4, November 13, 1980. (Refer



6. Radiation survey locations at Elevation 347 for Entry 4, November 13,1980. These survey points are on the in-core



7. Location of decontamination test area on Elevation 305 made during Entry 4, November 13,1980. The airlock in the figure is \#2 PAL 


\section{TABLES}

1. Task summary for TMI-2 Entry 4, November 13, $1980 \ldots \ldots \ldots \ldots \ldots$

2. Radiation survey, Elevation 305, November 13, $1980 \ldots \ldots \ldots \ldots \ldots$

3. Radiation survey, HPR-211 on Elevation 305 , November 13, $1980 \ldots 8$

4. Radiation survey, equipment hatch on Elevation 305, November $13,1980 \ldots \ldots \ldots \ldots \ldots \ldots \ldots \ldots \ldots \ldots \ldots \ldots \ldots \ldots . \ldots \ldots$

5. Radiation survey, Elevation 305 , November $13,1980 \ldots \ldots \ldots \ldots$

6. Radiation survey, Elevation 347 , November $13,1980 \ldots \ldots \ldots \ldots$

7. Radiation survey, Elevation 347 , November $13,1980 \ldots \ldots \ldots \ldots \ldots$

8. Surface contamination from decontamination test on

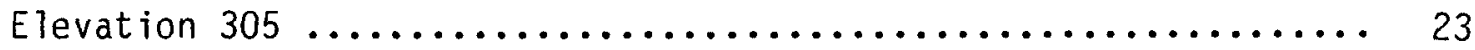

9. Airborne activity, November $13,1980 \ldots \ldots \ldots \ldots \ldots \ldots \ldots \ldots \ldots \ldots$ 


\section{ENTRY OVERVIEW}

Entry 4 into the reactor building at Three Mile Island Unit 2 occurred on November 13, 1980. Twelve technicians working in three teams entered containment at 20-minute intervals, taking with them both video and still cameras and radiation survey equipment.

Entry 4 yielded additional beta and gamma survey data at both Elevation 305 and Elevation 347. Locations for these measurements were selected based on observations of personnel involved in previous entries as well as on study of still photographs acquired during those entries.

On Elevation 305 a preliminary decontamination test was conducted. The test involved smear and scrape samples taken before, during, and after a demineralizing water wash, a Radiac wash, and a Radiac scrub.

Power receptacles were tested on Elevation 305 as well as on Elevation 347. Receptacles on the lower level are powered from Elevation 282 (the basement leve 1); these receptacles were all found to be deenergized. Receptacies on Elevation 347, on the other hand, were found to be carrying full power.

Data collected during entry 4 are presented in subsequent sections of this report. 
TASK SUMMARY

A variety of tasks was performed during entry 4. These tasks are summarized in Table 1. 
TABLE 1. TASK SUMMARY FOR TMI-2 ENTRY 4, NOVEMBER 13, 1980

Containment Temperature $69^{\circ}$ (approximately $20.5^{\circ} \mathrm{C}$ )

Containment Pressure $-0.2 \mathrm{in} . \mathrm{Hg}$

Relative Humidity $<100 \%$

Airborne Activity $<M P C$

\begin{tabular}{|c|c|c|c|c|}
\hline $\begin{array}{l}\text { Data Acquisition } \\
\text { Task Number } \\
\end{array}$ & $\begin{array}{c}\text { Task } \\
\text { Description }\end{array}$ & Task Accomplished & Problems Encountered & $\begin{array}{c}\text { Comments } \\
\text { Significant Findings }\end{array}$ \\
\hline $10 A$ & $\begin{array}{l}\text { Videotaped } \\
\text { Elevation } 305\end{array}$ & $\begin{array}{l}\text { Videotaped } \\
\text { Elevation } 305\end{array}$ & $\begin{array}{l}\text { Insufficient light } \\
\text { for high-quality } \\
\text { video }\end{array}$ & $\begin{array}{l}\text { Four low-light-level } \\
\text { cameras to be } \\
\text { installed on Eleva- } \\
\text { tion } 305 \text { in January- } \\
\text { February } 1981 .\end{array}$ \\
\hline $31 \mathrm{~A}, 31 \mathrm{~B}$ & $\begin{array}{l}\text { Videotaped } \\
\text { Elevation } 347\end{array}$ & $\begin{array}{l}\text { Videotaped } \\
\text { Elevation } 347\end{array}$ & $\begin{array}{l}\text { Insufficient light } \\
\text { for high-quality } \\
\text { video }\end{array}$ & $\begin{array}{l}\text { Better flood lights } \\
\text { to be installed } \\
\text { on future entries; } \\
\text { four low-light- } \\
\text { level cameras to be } \\
\text { installed on Eleva- } \\
\text { tion } 347 \text { in January- } \\
\text { February } 1981\end{array}$ \\
\hline$N / A$ & $\begin{array}{l}\text { Replace source } \\
\text { range preamp }\end{array}$ & Not performed & $\begin{array}{l}\text { Insufficient confi- } \\
\text { dence in system and } \\
\text { replacement part } \\
\text { warranted further } \\
\text { testing prior to } \\
\text { installation }\end{array}$ & $\begin{array}{l}\text { Installation to be } \\
\text { made in January } \\
1981\end{array}$ \\
\hline$N / A$ & $\begin{array}{l}\text { Still photographs } \\
\text { of Elevation } 347\end{array}$ & $\begin{array}{l}\text { Still photographs } \\
\text { of Elevation } 347\end{array}$ & None & None \\
\hline
\end{tabular}


TABLE 1. (continued)

\begin{tabular}{|c|c|c|c|c|}
\hline $\begin{array}{c}\text { Data Acquisition } \\
\text { Task Number } \\
\end{array}$ & $\begin{array}{c}\text { Task } \\
\text { Description } \\
\end{array}$ & Task Accomplished & Problems Encountered & $\begin{array}{c}\text { Comments } \\
\text { Significant Findings }\end{array}$ \\
\hline$N / A$ & $\begin{array}{l}\text { Investigate area } \\
\text { around Personnel } \\
\text { Airlock (PAL) \#2 for } \\
\text { for possible inter- } \\
\text { mediate containment }\end{array}$ & $\begin{array}{l}\text { Investigation } \\
\text { completed }\end{array}$ & None & None \\
\hline $4,6,122 \mathrm{~A}, 22 \mathrm{H}$ & $\begin{array}{l}\text { Conduct radiation } \\
\text { surveys }\end{array}$ & Surveys completed & None & $\begin{array}{l}\text { Improved survey of } \\
\text { the in-core } \\
\text { instrument table }\end{array}$ \\
\hline$N / A$ & $\begin{array}{l}\text { Move fuel crane and } \\
\text { assess access to } \\
\text { core flood tanks } \\
\text { from Elevation } 347\end{array}$ & $\begin{array}{l}\text { Fuel crane moved and } \\
\text { access to A-core } \\
\text { flood tank found } \\
\text { possible, B-core } \\
\text { flood tank very } \\
\text { difficult }\end{array}$ & None & None \\
\hline 42 & $\begin{array}{l}\text { Decontamination } \\
\text { experiment on } \\
\text { Elevation } 305 \text { floor }\end{array}$ & Experiment completed & None & None \\
\hline 38 & Paint chip samples & $\begin{array}{l}\text { Obtained paint chip } \\
\text { sample from reactor } \\
\text { building dome }\end{array}$ & None & None \\
\hline
\end{tabular}


Radiation was measured at many locations inside containment. Measurement locations, instruments used, and beta and gamma dose rates are presented in Table 2 through 7; survey points are indicated on Figures 1 through 6, which are associated with the tables.

Following these tables is a description of the decontamination test conducted during the entry; test location is shown in Figure 7 , and readings are given in Table 8 . Finally, Table 9 characterizes airborne activity. 
TABLE 2. RADIATION SURVEY, ELEVATION 305, NOVEMBER 13, 1980

\begin{tabular}{|c|c|c|c|c|c|}
\hline $\begin{array}{l}\text { Data Acquisition } \\
\text { Task Number } \\
\end{array}$ & $\begin{array}{l}\text { Location } \\
\text { Figure } 1^{\mathrm{a}} \\
\end{array}$ & Instrument & $\begin{array}{c}\text { Gamma } \\
\text { Dose Rate } \\
\text { (mrem/hr) }\end{array}$ & $\begin{array}{l}\text { Beta } \\
\text { Dose Rate } \\
\text { (rad/hr) } \\
\end{array}$ & Location \\
\hline 1 & 1 & $R 0-2 A$ & $450 \star b$ & $--c$ & South of PAL \#2 \\
\hline 1 & 2 & $R 0-2 A$ & 1200 & -- & Elevator door \\
\hline 1 & 3 & Ro-2A & $500 *$ & -- & In front of elevator \\
\hline 1 & 4 & $R 0-2 A$ & $600 *$ & -- & In front of air coolers \\
\hline 1 & 5 & $R 0-2 A$ & 800 & 9.2 & Floor in front of air coolers \\
\hline 1 & 6 & $\mathrm{R} 0-2 \mathrm{~A}$ & 620 & -- & South side of D-ring wall \\
\hline 4 & 7 & $R 0-2 A$ & 3800 & -- & B-core flood tank piping \\
\hline 6 & 8 & $R 0-2 A$ & 2000 & 9.9 & Floor drain \\
\hline 1 & 9 & $R 0-2 A$ & 600 & -- & Near PAL \#1 \\
\hline 1 & 10 & $R O-2 A$ & 500 & 3.3 & Floor in front of PAL \#1 \\
\hline 1 & 11 & $\mathrm{RO}-2 \mathrm{~A}$ & $600 *$ & -- & North side of A-core flood tank \\
\hline 1 & 12 & $R 0-2 A$ & 400 & -- & D-ring near open stairwell \\
\hline 1 & 13 & $R 0-2 A$ & 3000 & 16.5 & Chains on floor near open stairwell \\
\hline 1 & 14 & $R 0-2 A$ & 1000 & -- & Near open stairwell \\
\hline 1 & 15 & $R O-2 A$ & $300 *$ & -- & Northwest section of reactor building \\
\hline 1 & 16 & $R O-2 A$ & $280-390$ & -- & Access fence to in-core chase \\
\hline
\end{tabular}


TABLE 2. (continued)

\begin{tabular}{|c|c|c|c|c|c|}
\hline $\begin{array}{l}\text { Data Acquisition } \\
\text { Task Number } \\
\end{array}$ & $\begin{array}{l}\text { Location } \\
\text { Figure } 1^{\mathrm{a}} \\
\end{array}$ & Instrument & $\begin{array}{c}\text { Gamma } \\
\text { Dose Rate } \\
\text { (mrem/hr) } \\
\end{array}$ & $\begin{array}{l}\text { Beta } \\
\text { Dose Rate } \\
\text { (rad/hr) } \\
\end{array}$ & Location \\
\hline 6 & 17 & $R 0-2 A$ & 2000 & 26.4 & Floor drain \\
\hline 1 & 18 & $\mathrm{RO}-2 \mathrm{~A}$ & 1400 & $--c$ & Floor penetration on west side of D-ring \\
\hline 1 & 19 & $R 0-2 A$ & 1200 & -- & Floor penetration on west side of D-ring \\
\hline 1 & 20 & $R O-2 A$ & $800 *$ & -- & $\begin{array}{l}\text { Seismic gap on east side of reactor } \\
\text { building }\end{array}$ \\
\hline 1 & 21 & $R O-2 A$ & $200 *$ & -- & Northeast section of reactor building \\
\hline 6 & 22 & $\mathrm{RO}-2 \mathrm{~A}$ & 600 & 4.6 & Floor drain \\
\hline
\end{tabular}

a. Figure 1 follows the table.

b. The asterisk (*) denotes general area readings--all others are contact readings.

c. The dash (--) indicates no reading taken. 
TABLE 3. RADIATION SURVEY, HPR-211 ON ELEVATION 305, NOVEMBER 13, 1980

\begin{tabular}{|c|c|c|c|c|c|}
\hline $\begin{array}{l}\text { Data Acquisition } \\
\text { Task Number } \\
\end{array}$ & $\begin{array}{l}\text { Location } \\
\text { Figure } 2^{a} \\
\end{array}$ & Instrument & $\begin{array}{l}\text { Gamma } \\
\text { Dose Rate } \\
\text { (mrem/hr) } \\
\end{array}$ & $\begin{array}{l}\text { Beta } \\
\text { Dose Rate } \\
(\text { rad/hr) } \\
\end{array}$ & Location \\
\hline 1 & 1 & $R O-2 A$ & 250 & 0.165 & $\begin{array}{l}\text { Just above HPR-211 mounting bar } \\
\text { (contact) }\end{array}$ \\
\hline 1 & 2 & $R 0-2 A$ & 250 & 0.033 & $\begin{array}{l}\text { Just below HPR-211 mounting bar } \\
\text { (contact) }\end{array}$ \\
\hline 1 & 3 & $\mathrm{RO}-2 \mathrm{~A}$ & 400 & $--b$ & $\begin{array}{l}\text { Area around } H P R-211 \text { at } 1-\mathrm{ft} \text { (approxi- } \\
\text { mately } 30.5 \mathrm{~cm} \text { ) radius and } 1 \mathrm{ft} \text { from } \\
\text { wal1 }\end{array}$ \\
\hline 1 & 4 & $\mathrm{RO}-2 \mathrm{~A}$ & 280 & -- & $\begin{array}{l}\text { Area around HPR-211 at } 1-\mathrm{ft} \text { (approxi- } \\
\text { mately } 30.5 \mathrm{~cm} \text { ) radius and } 1 \mathrm{ft} \text { from } \\
\text { wal1 }\end{array}$ \\
\hline 1 & 5 & $\mathrm{RO}-2 \mathrm{~A}$ & 350 & -- & $\begin{array}{l}\text { Area around } H P R-211 \text { at } 1-\mathrm{ft} \text { (approxi- } \\
\text { mately } 30.5 \mathrm{~cm} \text { ) radius and } 1 \mathrm{ft} \text { from } \\
\text { wa } 11\end{array}$ \\
\hline 1 & 6 & $R O-2 A$ & 480 & -- & $\begin{array}{l}\text { Area around HPR-211 at } 1-\mathrm{ft} \text { (approxi- } \\
\text { mately } 30.5 \mathrm{~cm} \text { ) radius and } 1 \mathrm{ft} \text { from } \\
\text { wal1 }\end{array}$ \\
\hline 1 & 7 & $\mathrm{RO}-2 \mathrm{~A}$ & 400 & - & $\begin{array}{l}\text { Area around HPR-211 at 1-ft (approxi- } \\
\text { mately } 30.5 \mathrm{~cm} \text { ) radius and } 1 \mathrm{ft} \text { from } \\
\text { wal1 }\end{array}$ \\
\hline
\end{tabular}

a. Figure 2 follows the table.

b. The dash (--) indicates no reading taken. 
TABLE 4. RADIATION SURVEY EQUIPMENT HATCH ON ELEVATION 305, NOVEMBER 13,1980

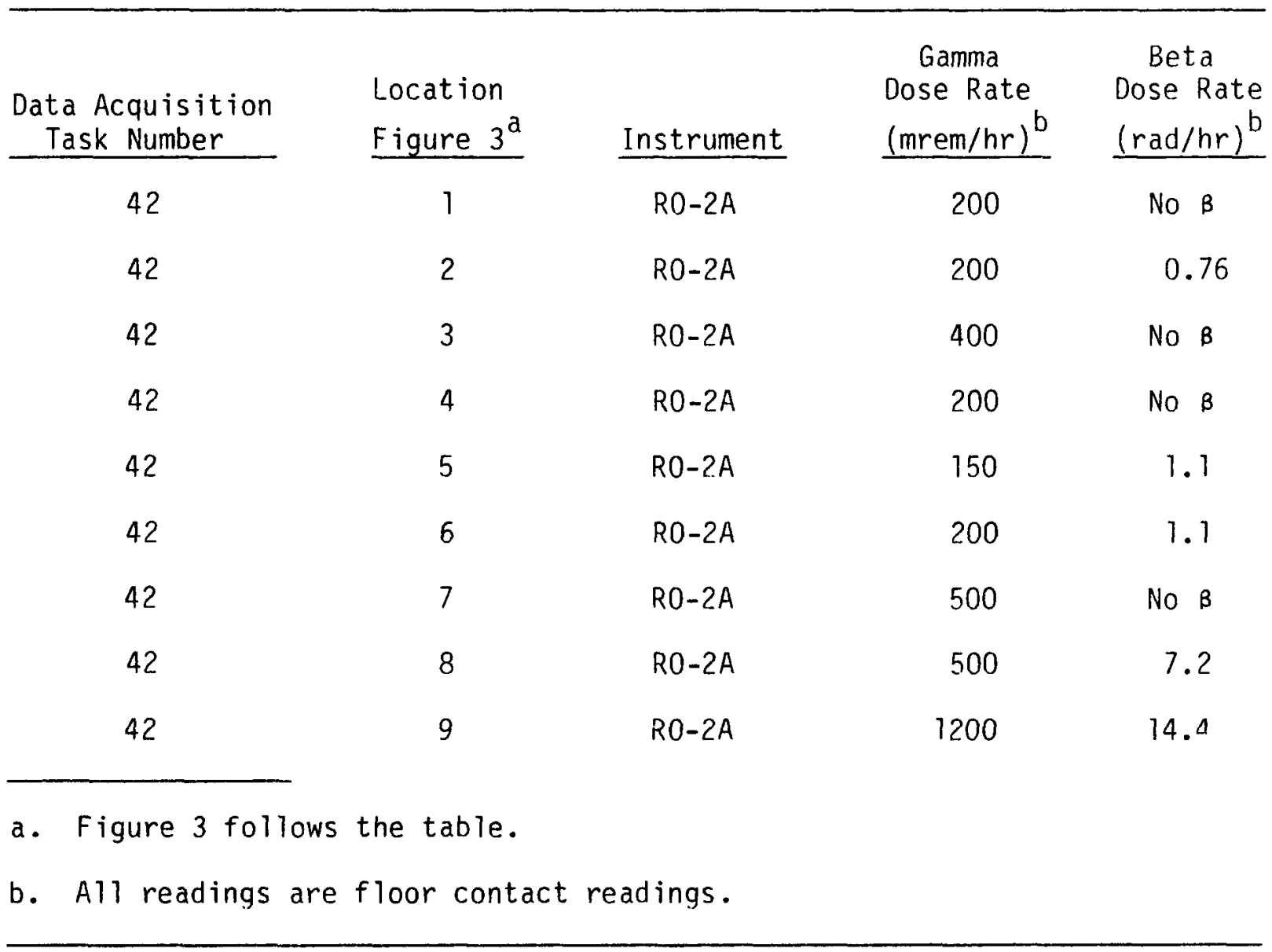


TABLE 5. RADIATION SURVEY, ELEVATION 305, NOVEMBER 13, 1980

\begin{tabular}{|c|c|c|c|c|c|}
\hline $\begin{array}{l}\text { Data Acquisition } \\
\text { Task Number } \\
\end{array}$ & $\begin{array}{l}\text { Location } \\
\text { Figure } 4^{\mathrm{a}} \\
\end{array}$ & Instrument & $\begin{array}{c}\text { Gamma } \\
\text { Dose Rate } \\
(\text { mrem } / h r) \\
\end{array}$ & $\begin{array}{l}\text { Beta } \\
\text { Dose Rate } \\
(\mathrm{rad} / \mathrm{hr}) \\
\end{array}$ & Location \\
\hline 42 & 1 & $\mathrm{RO}-2 \mathrm{~A}$ & 200 & 3.8 & See Figure 4 \\
\hline 42 & 2 & $R O-2 A$ & 200 & 3.8 & See Figure 4 \\
\hline 42 & 3 & $\mathrm{R} 0-2 \mathrm{~A}$ & 500 & 3.8 & See Figure 4 \\
\hline 42 & 4 & $R O-2 A$ & 500 & 4.5 & See Figure 4 \\
\hline 42 & 5 & $R 0-2 A$ & 500 & 4.9 & See Figure 4 \\
\hline 42 & 6 & $R O-2 A$ & 400 & 4.2 & See Figure 4 \\
\hline 42 & 7 & $\mathrm{RO}-2 \mathrm{~A}$ & 300 & 3.4 & See Figure 4 \\
\hline 42 & 8 & $\mathrm{RO}-2 \mathrm{~A}$ & 200 & 1.1 & See Figure 4 \\
\hline 42 & 9 & $R O-2 A$ & 500 & 3.9 & See Figure 4 \\
\hline 42 & 10 & $\mathrm{RO}-2 \mathrm{~A}$ & 200 & 5.3 & See Figure 4 \\
\hline 42 & 11 & $R O-2 A$ & 300 & 4.6 & See Figure 4 \\
\hline 42 & 12 & $\mathrm{RO}-2 \mathrm{~A}$ & 500 & 5.7 & See Figure 4 \\
\hline 42 & 13 & $\mathrm{RO}-2 \mathrm{~A}$ & 400 & 3.8 & See Figure 4 \\
\hline 42 & 14 & $\mathrm{RO}-2 \mathrm{~A}$ & 500 & 3.8 & Prior to decontamination test operations \\
\hline 42 & 14 & $\mathrm{R} 0-2 \mathrm{~A}$ & 400 & 2.3 & After water wash \\
\hline 42 & 14 & $R 0-2 A$ & 400 & 2.3 & After Radiac wash \\
\hline
\end{tabular}


TABLE 5. (cont inued)




TABLE 6. RADIATION SURVEY, ELEVATION 347, NOVEMBER 13, 1980

\begin{tabular}{|c|c|c|c|c|c|}
\hline $\begin{array}{l}\text { Data Acquisition } \\
\text { Task Number } \\
\end{array}$ & $\begin{array}{l}\text { Location } \\
\text { Figure } 5^{a} \\
\end{array}$ & Instrument & $\begin{array}{c}\text { Gamma } \\
\text { Dose Rate } \\
\text { (mrem/hr) }\end{array}$ & $\begin{array}{l}\text { Beta } \\
\text { Dose Rate } \\
(\mathrm{rad} / \mathrm{hr}) \\
\end{array}$ & Location \\
\hline $22 \mathrm{~A}$ & 1 & Teletector & $200 * b$ & $--c$ & Inside of elevator shaft \\
\hline $22 \mathrm{~A}$ & 2 & $R O-2 A$ & $500 *$ & -- & Southside of enclosed stairwell \\
\hline $22 \mathrm{~A}$ & 3 & $R 0-2 A$ & $500 *$ & -- & $\begin{array}{l}\text { Northeast corner of equipment hatch } \\
\text { deck plate }\end{array}$ \\
\hline $22 \mathrm{~A}$ & 4 & $\mathrm{RO}-2 \mathrm{~A}$ & $500 *$ & -- & West side of equipment hatch deck plate \\
\hline $22 A$ & 5 & $R 0-2 A$ & $500^{\star}$ & -- & $\begin{array}{l}\text { Southwest corner of equipment hatch } \\
\text { deck plate }\end{array}$ \\
\hline $22 A$ & 6 & $\mathrm{RO}-2 \mathrm{~A}$ & $500^{\star}$ & -- & B-core flood tank grating \\
\hline $22 \mathrm{~A}$ & 7 & $R 0-2 A$ & $350^{\star}$ & & First landing of open stairwell \\
\hline $22 A$ & 8 & $R 0-2 A$ & 300 & $5.6 *$ & Gap between stairs of open stairwell \\
\hline $22 \mathrm{H}$ & 9 & $R O-2 A$ & 1000 & 9.9 & Floor drain \\
\hline $22 \mathrm{~A}$ & 10 & $R O-2 A$ & $100 *$ & -- & Near deck plate for A-core flood tank \\
\hline $22 \mathrm{~A}$ & 11 & $\mathrm{RO}-2 \mathrm{~A}$ & $150^{\star}$ & -- & Deck plate over A-core flood tank \\
\hline $22 \mathrm{~A}$ & 12 & $R O-2 A$ & $200 *$ & -- & East of A-core flood tank \\
\hline $22 \mathrm{H}$ & 13 & $\mathrm{RO} 0-2 \mathrm{~A}$ & $350^{\star}$ & 2.2 & Floor drain \\
\hline $22 \mathrm{~A}$ & 14 & $R O-2 A$ & $150 *$ & -- & Second landing of open stairwell \\
\hline
\end{tabular}


TABLE 6. (continued)

\begin{tabular}{|c|c|c|c|c|c|}
\hline $\begin{array}{l}\text { Data Acquisition } \\
\text { Task Number } \\
\end{array}$ & $\begin{array}{l}\text { Location } \\
\text { Figure } 5^{\text {a }} \\
\end{array}$ & Instrument & $\begin{array}{c}\text { Gamma } \\
\text { Dose Rate } \\
\text { (mrem } / \mathrm{hr} \text { ) }\end{array}$ & $\begin{array}{l}\quad \text { Beta } \\
\text { Dose Rate } \\
\text { (rad/hr) } \\
\end{array}$ & Location \\
\hline $22 \mathrm{~A}$ & 15 & $\mathrm{RO}-2 \mathrm{~A}$ & $100^{*}$ & -- & $\begin{array}{l}\text { Top landing of open stairwell } \\
\text { (Elevation } 367 \text { ) }\end{array}$ \\
\hline $22 \mathrm{~A}$ & 16 & $\mathrm{R} 0-2 \mathrm{~A}$ & $100 *$ & $0.594 *$ & Top of east D-ring (Elevation 367) \\
\hline $22 \mathrm{~A}$ & 17 & $\mathrm{RO}-2 \mathrm{~A}$ & $100 *$ & $0.594 *$ & Top of east D-ring (Elevation 367) \\
\hline $22 \mathrm{~A}$ & 18 & $\mathrm{RO}-2 \mathrm{~A}$ & $200 *$ & $0.825 *$ & $\begin{array}{l}\text { Arm's reach into east D-ring (Elevation } \\
367 \text { ) }\end{array}$ \\
\hline $22 \mathrm{~A}$ & 19 & $R 0-2 A$ & 300 & 8.9 & $\begin{array}{l}\text { Top of steel support beam (Elevation } \\
367 \text { ) }\end{array}$ \\
\hline $22 \mathrm{~A}$ & 20 & $\mathrm{RO} 0-2 \mathrm{~A}$ & $100 *$ & $0.594 *$ & $\begin{array}{l}\text { Top of D-ring on south side (Elevation } \\
367 \text { ) }\end{array}$ \\
\hline $22 \mathrm{~A}$ & 21 & $\mathrm{RO} 0-2 \mathrm{~A}$ & $600 *$ & $1.650 *$ & $\begin{array}{l}\text { Arm's reach into east Daring (Elevation } \\
367 \text { ) }\end{array}$ \\
\hline $22 \mathrm{~A}$ & 22 & $\mathrm{R} 0-2 \mathrm{~A}$ & $150 *$ & $0.165^{\star}$ & $\begin{array}{l}\text { South walkway between D-rings } \\
\text { (Elevation } 367 \text { ) }\end{array}$ \\
\hline $22 \mathrm{~A}$ & 23 & $\mathrm{R} 0-2 \mathrm{~A}$ & $700 *$ & $0.330^{\star}$ & $\begin{array}{l}\text { Arm's reach into west D-ring (Elevation } \\
367 \text { ) }\end{array}$ \\
\hline $22 \mathrm{~A}$ & 24 & $\mathrm{R} 0-2 \mathrm{~A}$ & 250 & 9 & $\begin{array}{l}\text { Top of steel support ring (Elevation } \\
367 \text { ) }\end{array}$ \\
\hline $22 \mathrm{~A}$ & 25 & $R 0-2 A$ & $500 *$ & No $\beta^{*}$ & $\begin{array}{l}\text { Arm's reach into west D-ring (Elevation } \\
367 \text { ) }\end{array}$ \\
\hline
\end{tabular}


TABLE 6. (continued)

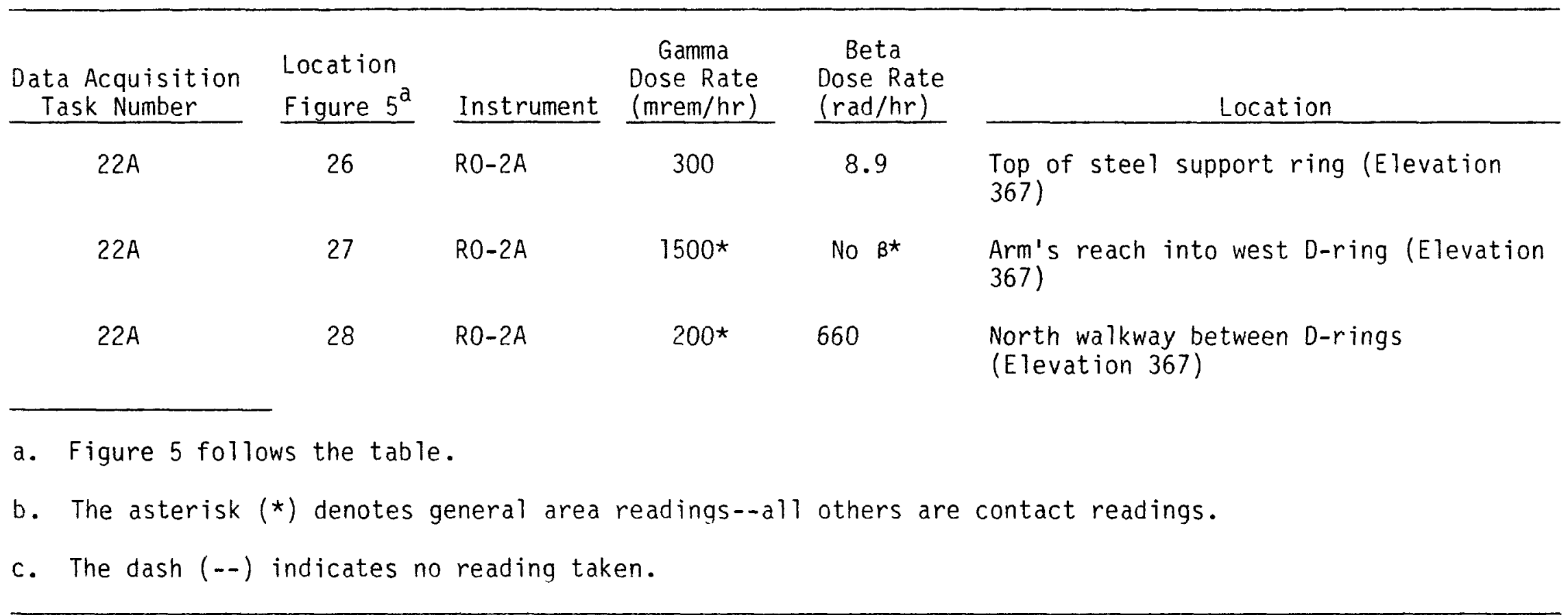


TABLE 7. RADIATION SURVEY, ELEVATION 347, NOVEMBER 13, 1980

\begin{tabular}{|c|c|c|c|c|}
\hline $\begin{array}{l}\text { Data Acquisition } \\
\text { Task Number } \\
\end{array}$ & $\begin{array}{l}\text { Location } \\
\text { Figure } 6^{\mathrm{a}} \\
\end{array}$ & Instrument & $\begin{array}{l}\text { Gamma } \\
\text { Dose Rate } \\
\text { (mrem/hr) } \\
\end{array}$ & $\begin{array}{l}\text { Beta } \\
\text { Dose Rate } \\
(\mathrm{rad} / \mathrm{hr}) \\
\end{array}$ \\
\hline $\begin{array}{l}22 \mathrm{H} \\
22 \mathrm{H}\end{array}$ & 1 & $\begin{array}{l}R 0-2 A \\
R 0-2 A\end{array}$ & $\begin{array}{l}5,000 * b \\
5,000\end{array}$ & $>149^{4.95^{\star}}$ \\
\hline $\begin{array}{l}22 \mathrm{H} \\
22 \mathrm{H}\end{array}$ & 2 & $\begin{array}{l}R 0-2 A \\
R 0-2 A\end{array}$ & $\begin{array}{l}1,500^{\star} \\
10,000\end{array}$ & $>11.95^{\star}$ \\
\hline $\begin{array}{l}22 \mathrm{H} \\
22 \mathrm{H}\end{array}$ & 3 & $\begin{array}{l}\mathrm{RO}-2 \mathrm{~A} \\
\mathrm{R} 0-2 \mathrm{~A}\end{array}$ & $\begin{array}{l}10,000 * \\
15,000\end{array}$ & $\begin{aligned} & 16.5^{*} \\
> & 115.5\end{aligned}$ \\
\hline $\begin{array}{l}22 \mathrm{H} \\
22 \mathrm{H}\end{array}$ & 4 & $\begin{array}{l}R 0-2 A \\
R 0-2 A\end{array}$ & $\begin{array}{l}1,800 \star \\
2,000\end{array}$ & $\begin{array}{l}10.5 \star \\
9.9\end{array}$ \\
\hline $\begin{array}{l}22 \mathrm{H} \\
22 \mathrm{H}\end{array}$ & 5 & $\begin{array}{l}R 0-2 A \\
R 0-2 A\end{array}$ & $\begin{array}{c}10,000 \star \\
3,500\end{array}$ & $\begin{aligned} & 13.5 * \\
> & 153.4\end{aligned}$ \\
\hline $\begin{array}{l}\text { a. Figure } 6 \text { foll } \\
\text { b. The asterisk } \\
\text { readings. }\end{array}$ & $\begin{array}{l}\text { the table. } \\
\text { denotes wai }\end{array}$ & vel readir & 11 others & contact \\
\hline
\end{tabular}




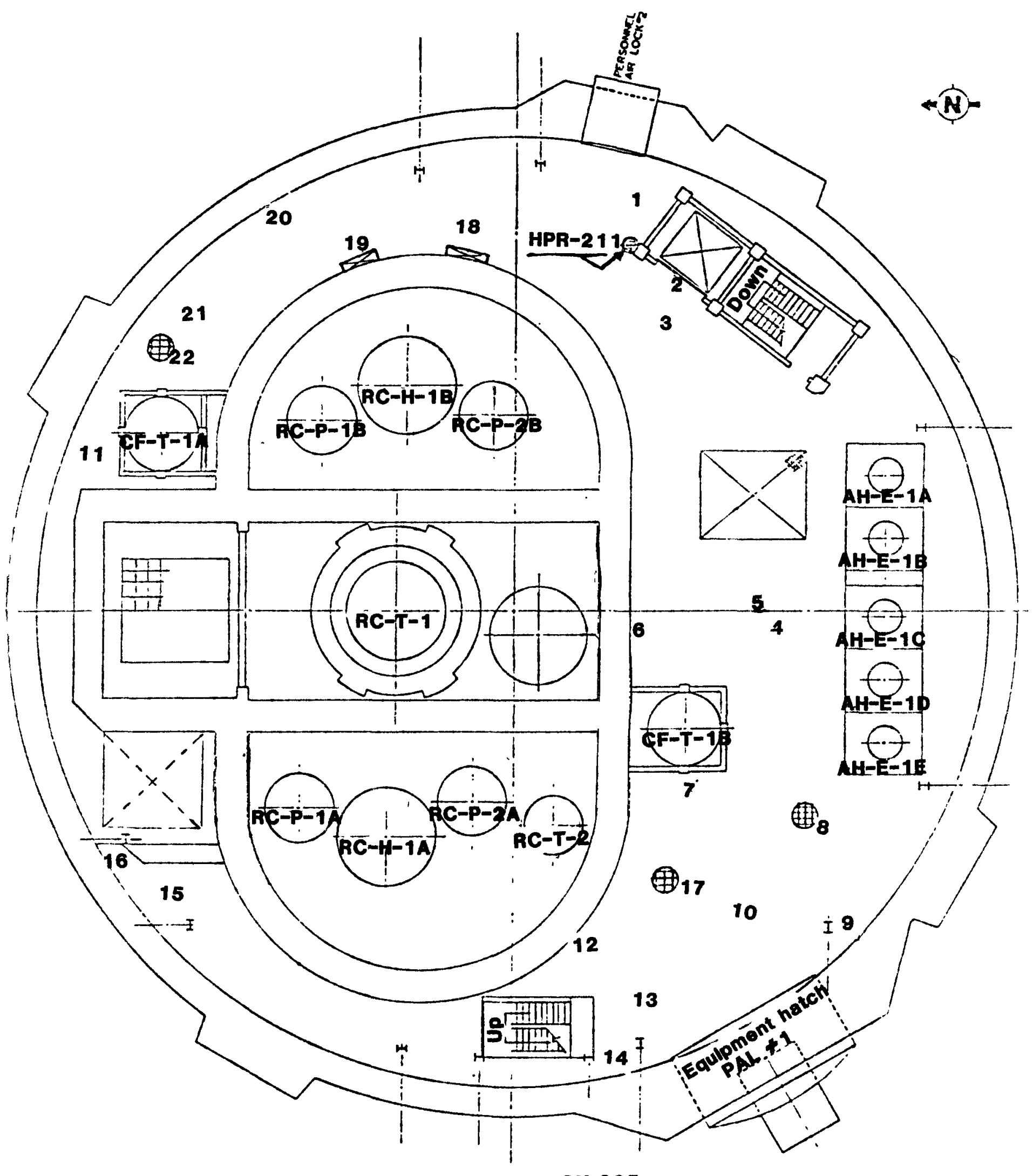

PLAN VIEW, ELEVATION 305

Figure 1. Radiation survey locations on Elevation 305 for Entry 4 , November 13, 1980. (Refer to Table 2.) 




$$
\begin{gathered}
\text { HPR-21l Area Survey } \\
\text { Located on Closed Stairwell wall (See Figure 1) } \\
\text { Radiation Survey Points } \\
\text { November 13, } 1980 \text { Entry }
\end{gathered}
$$

Figure 2. Radiation survey location on Elevation 305 for Entry 4, November 13, 1980. This is the HPR-211 area survey, located on the enclosed stairwell wall, as indicated in Figure 1. (Refer to Table 3.) 


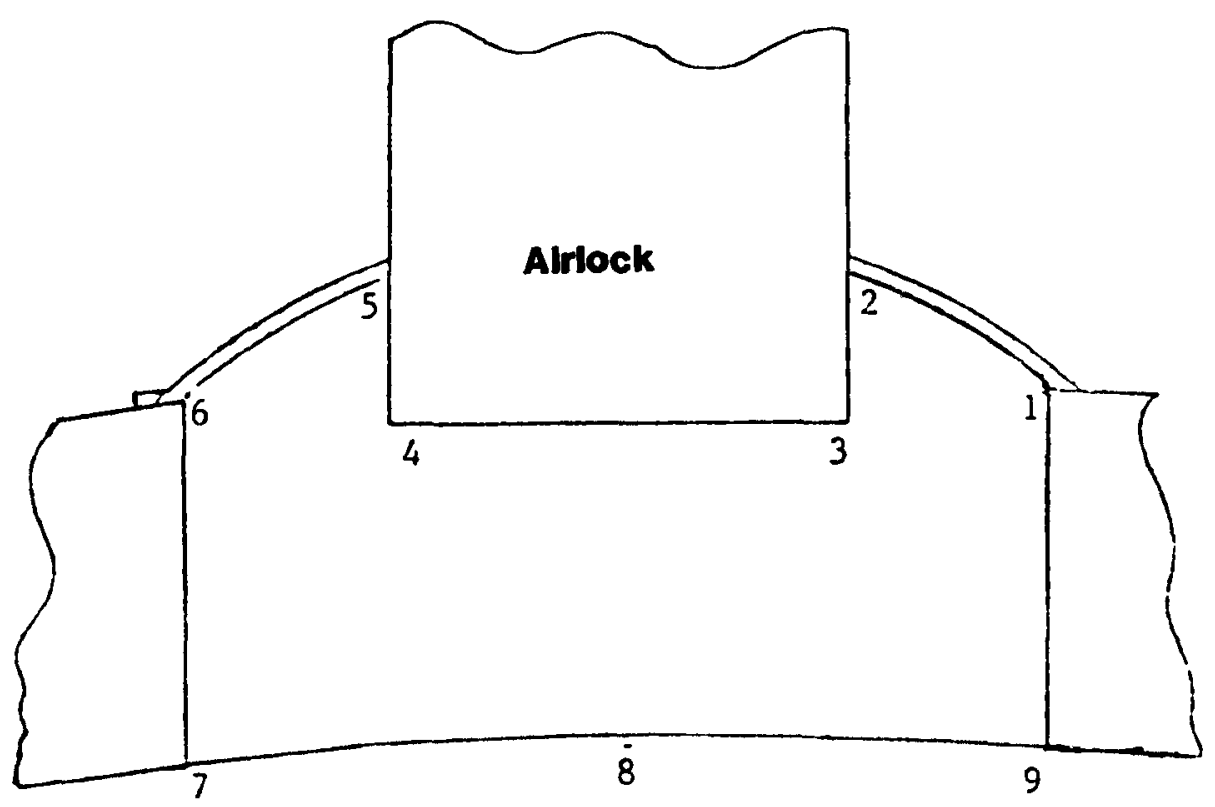

Reactor Building Equipment Hatch

Plan View

Radiation Survey Points

November 13, 1980 Entry

Figure 3. Radiation survey locations on Elevation 305 for Entry 4, November 13, 1980. These survey locations are indicated on the plan view of the reactor building equipment hatch. (Refer to Table 4.) 


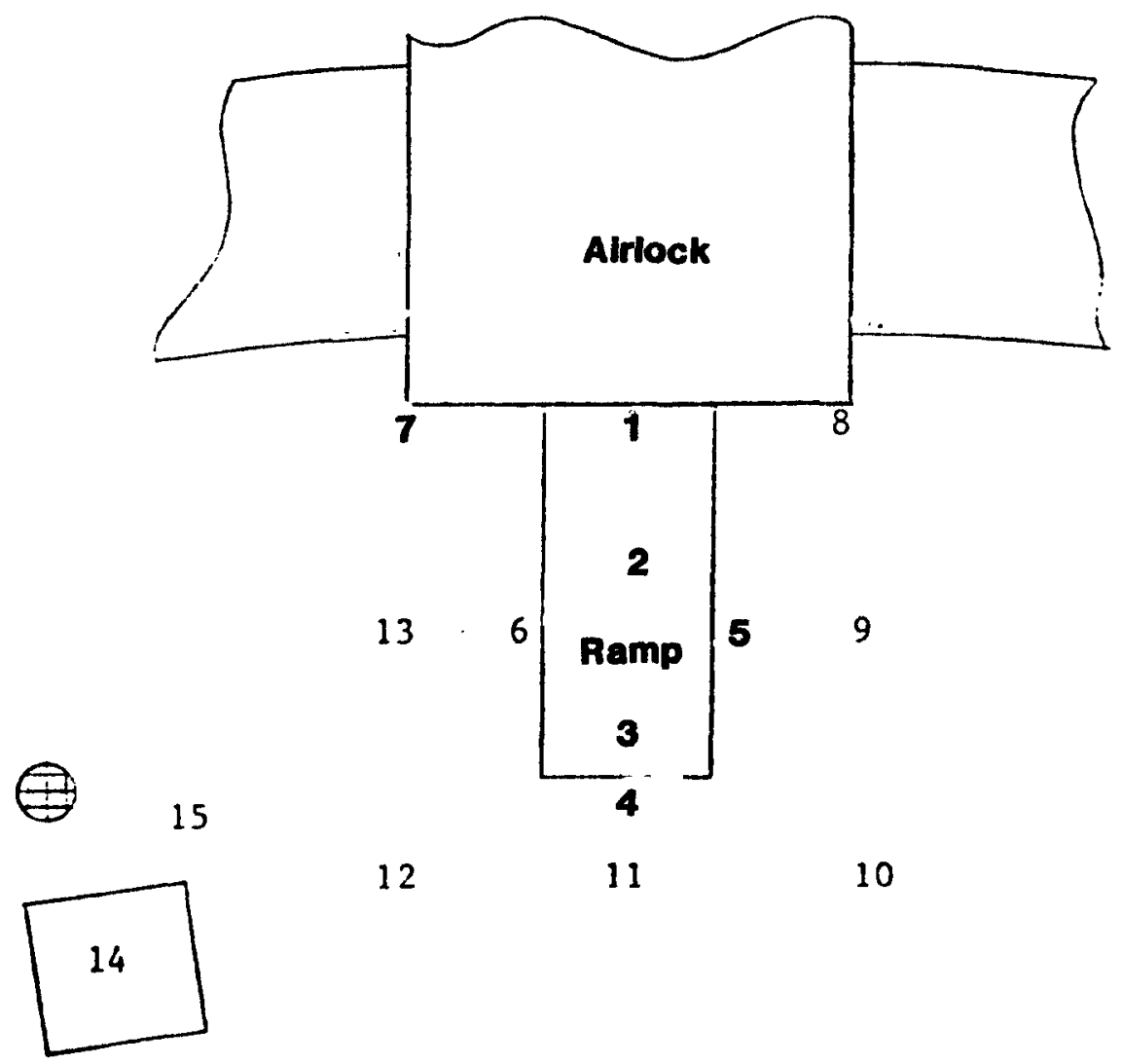

Reactor Building Personnel Airlock \#2

$$
\begin{aligned}
& \text { Plan View } \\
& \text { Radiation Survey Points } \\
& \text { November 13, } 1980 \text { Entry }
\end{aligned}
$$

Figure 4. Radiation survey locations on Elevation 305 for Entry 4 , November 13,1980. These survey points are indicated on the plan view of the reactor building Personnel Airlock (PAL) \#2. (Refer to Table 5.) 


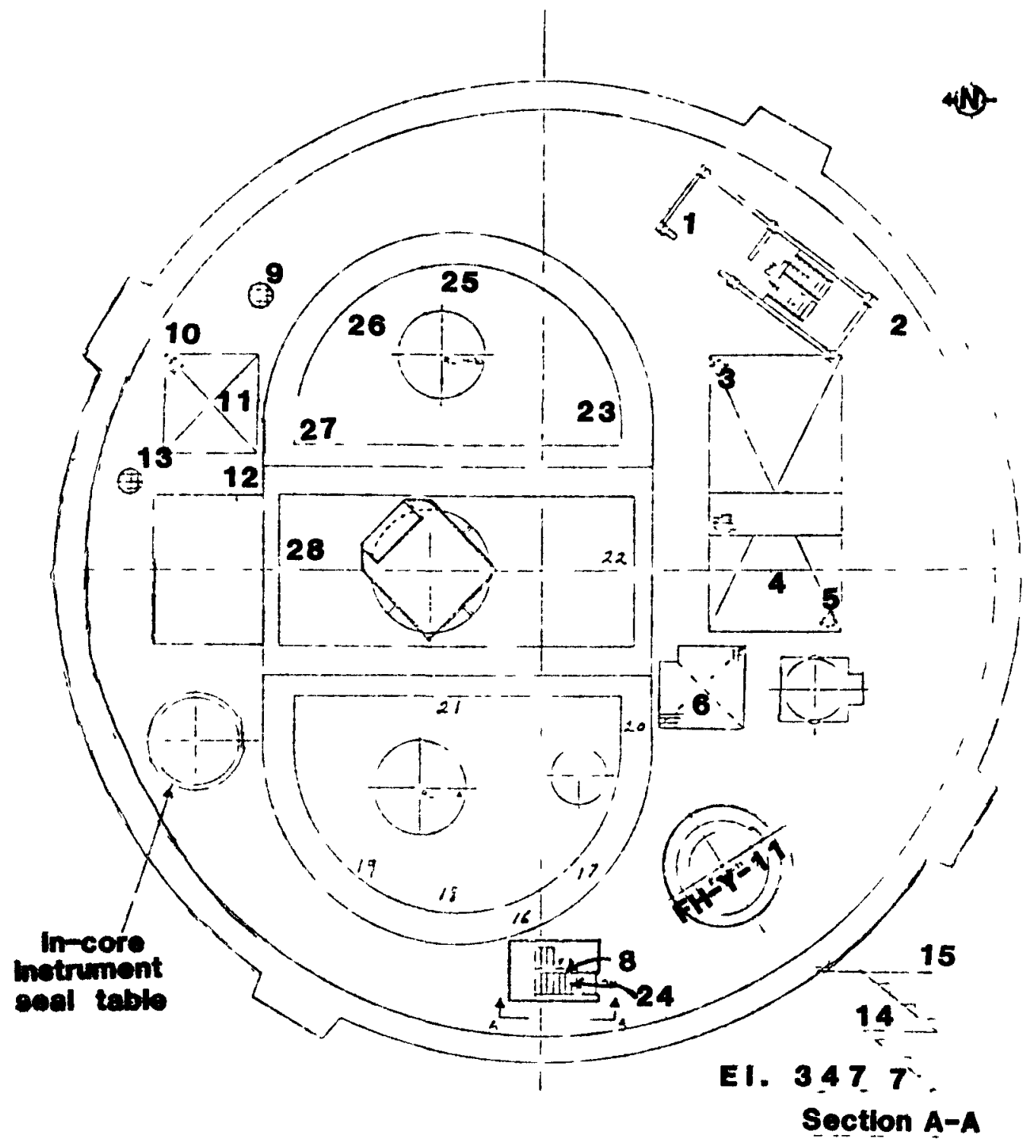

PLAN EL 347'-6"

Radiation Survey Points November 13, 1980 Entry

(Data points 16 through 28 a El. 367')

Figure 5. Radiation survey location on Elevation 305 (data points 16-28 are at Elevation 367) for Entry 4, November 13, 1980. (Refer to Table 6.) 


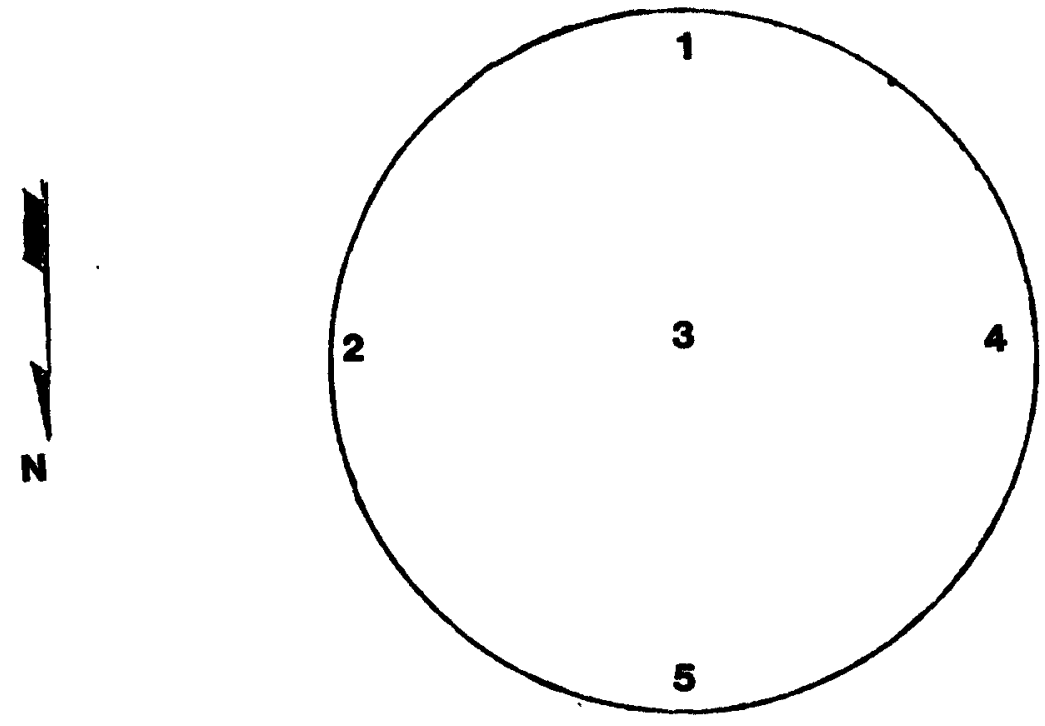

\section{Incore Instrumentation Seal Table \\ Plan View (See Figure 5) \\ Radiation Survey Points \\ November 13, 1980 Entry}

Figure 6. Radiation survey locations at Elevation 347 for Entry 4, November 13,1980. These survey points are on the in-core instrumentation seal tabel. (Refer to Table 7.) 
Smear samples 1 through 8 were taken in the center of a two foot by two foot area. This area is located on Elevation 305' between the \#2 PAL entry ramp and the D-ring as shown below. Sequence of the smears was as follows:



Scrape Sample 9 was taken outside the decon area. Scrape Sample 10 was taken in the decon area after the final smears.

Speciman 11 is a paint chip found on the floor at the $347^{\prime}$ Elevation. It came from the dome of the reactor building (approximately $300 \mathrm{~cm}^{2}$ ).

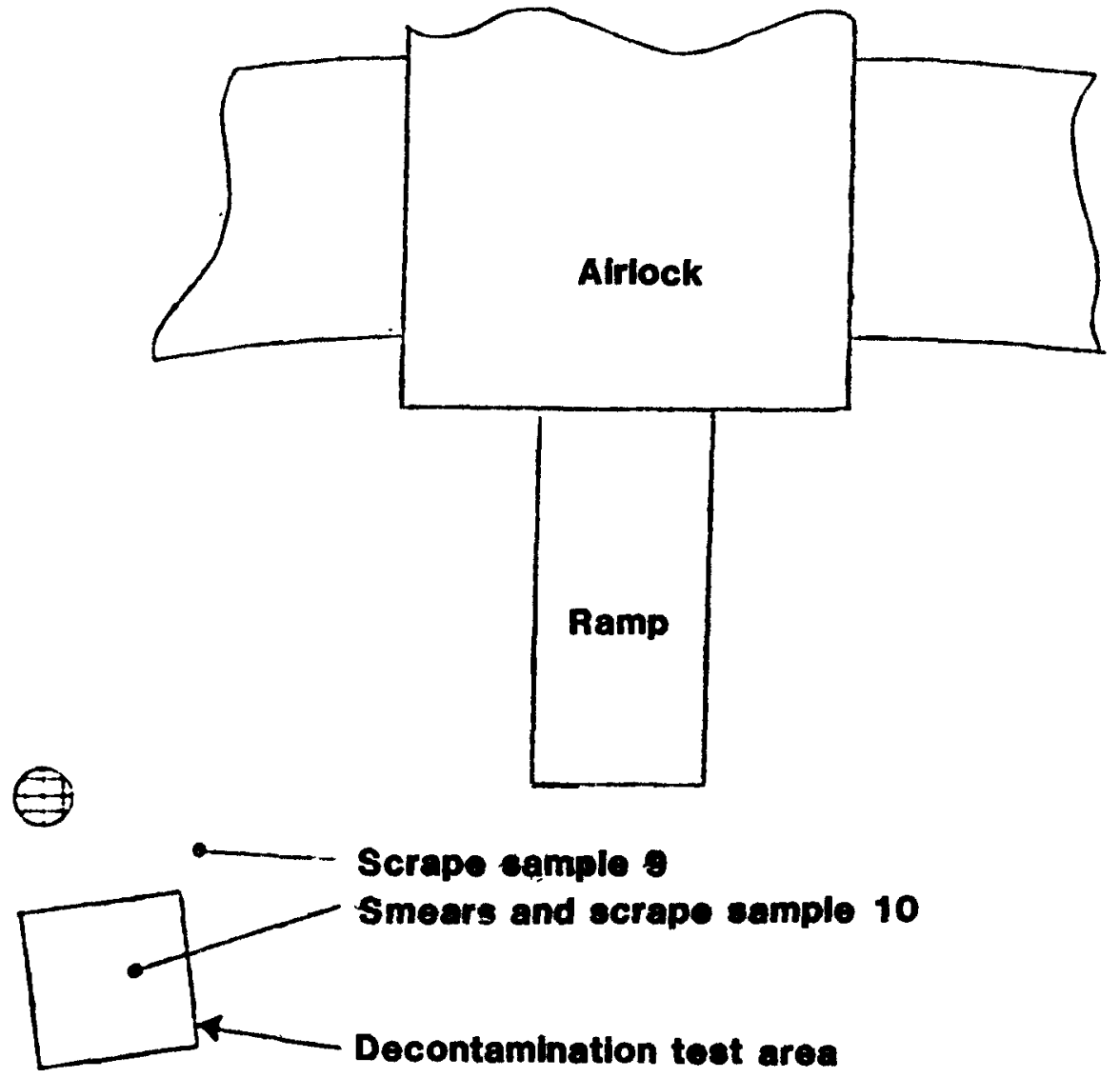

Reactor Building Personnel Airlock \#2

Figure 7. Location of decontamination test area on Elevation 305 made during Entry 4, November 13,1980. The airlock in the figure is \#2 PAL. 
TABLE 8. SURFACE CONTAMINATION FROM DECONTAMINATION TEST ON ELEVATION 305

\begin{tabular}{|c|c|c|c|c|c|c|c|}
\hline $\begin{array}{c}\text { Data } \\
\text { Acquisition } \\
\text { Task Number } \\
\end{array}$ & Specimen $^{a}, b, c$ & $\begin{array}{l}\text { Gross } \\
\text { Sample } \\
\text { Number } \\
\end{array}$ & $\begin{array}{l}\text { Gross alpha } \\
(\mu C i)(B \& W)^{d, e}\end{array}$ & $\begin{array}{l}\text { Gross } \\
\text { beta \& gamma } \\
(\mu C i)(B \& W)^{d, e}\end{array}$ & $\begin{array}{l}S r^{90} / \gamma^{90} \\
(\mu C i) \\
(S A I)^{d, f} \\
\end{array}$ & $\begin{array}{c}C^{134} \\
(\mu C i) \\
(B \& W / S A I)^{e, f} \\
\end{array}$ & $\begin{array}{c}C s^{137} \\
(\mu C i) \\
(B \& W / S A I)^{e, f} \\
\end{array}$ \\
\hline 42 & 1 & 52503 & $\begin{array}{l}-- \\
--\end{array}$ & -- & $2.3 E-1$ & $\begin{array}{l}1.7 \mathrm{E}+0 / \\
1.0 \mathrm{E}+0\end{array}$ & $\begin{array}{l}1.2 E+1 / \\
6.4 E+0\end{array}$ \\
\hline 42 & 2 & 52504 & -- & -- & $5.7 E-1$ & $\begin{array}{l}1.3 E+0 \\
7.0 E-1\end{array}$ & $\begin{array}{l}8.9 E+0 / \\
4.2 E+0\end{array}$ \\
\hline 42 & 3 & 52505 & -- & - & $5.2 E-3$ & $\begin{array}{l}1.8 \mathrm{E}-2 / \\
1.4 \mathrm{E}-2\end{array}$ & $\begin{array}{l}1.4 \mathrm{E}-1 / \\
1.1 \mathrm{E}-1\end{array}$ \\
\hline 42 & 4 & 52506 & -- & -- & $1.4 \mathrm{E}-2$ & $\begin{array}{l}2.6 \mathrm{E}-2 / \\
2.1 \mathrm{E}-2\end{array}$ & $\begin{array}{l}1.9 \mathrm{E}-1 / \\
1.6 \mathrm{E}-1\end{array}$ \\
\hline 42 & 5 & 52507 & $<2.7 E-7 g$ & $3.2 \mathrm{E}-2$ & $5.6 \mathrm{E}-3$ & $\begin{array}{l}7.8 \mathrm{E}-3 / \\
7.6 \mathrm{E}-3\end{array}$ & $\begin{array}{l}5.8 \mathrm{E}-2 / \\
5.3 \mathrm{E}-2\end{array}$ \\
\hline 42 & 6 & 52508 & $<2.7 E-79$ & $3.4 E-2$ & $7.7 \mathrm{E}-4$ & $\begin{array}{l}3.8 \mathrm{E}-3 / \\
1.8 \mathrm{E}-3\end{array}$ & $\begin{array}{l}2.8 \mathrm{E}-2 / \\
1.3 \mathrm{E}-2\end{array}$ \\
\hline 42 & 7 & 52509 & $<2.7 E-79$ & $8.7 E-3$ & $3.3 E-3$ & $\begin{array}{l}8.5 \mathrm{E}-3 / \\
5.8 \mathrm{E}-3\end{array}$ & $\begin{array}{l}6.3 \mathrm{E}-2 / \\
4.1 \mathrm{E}-2\end{array}$ \\
\hline 42 & 8 & 52510 & $<2.7 E-79$ & $1.0 E-2$ & $1.2 E-3$ & $\begin{array}{l}5.1 \mathrm{E}-3 / \\
2.2 \mathrm{E}-3\end{array}$ & $\begin{array}{l}3.6 \mathrm{E}-2 / \\
1.5 \mathrm{E}-2\end{array}$ \\
\hline 42 & 9 & 52511 & -- & -- & -- & $3.4 \mathrm{E}-1 /$ & $2.4 E+0 /$ \\
\hline
\end{tabular}


TABLE 8. (continued)

\begin{tabular}{|c|c|c|c|c|c|c|c|}
\hline $\begin{array}{l}\quad \text { Data } \\
\text { Acquisition } \\
\text { Task Number } \\
\end{array}$ & Specimen $^{a, b, c}$ & $\begin{array}{l}\text { Gross } \\
\text { Sample } \\
\text { Number }\end{array}$ & $\begin{array}{l}\text { Gross alpha } \\
(\mu C i)(B \& W)^{d, e} \\
\end{array}$ & $\begin{array}{l}\text { Gross } \\
\text { beta \& gamma } \\
(\mu \mathrm{C} \mathrm{j})(\mathrm{B} \& W)^{d, e} \\
\end{array}$ & $\begin{array}{l}S r^{90} / \gamma^{90} \\
(\mu C i) \\
(S A I)^{d, f} \\
\end{array}$ & $\begin{array}{c}C s^{134} \\
(\mu C i) \\
(B \& W / S A I)^{e, f} \\
\end{array}$ & $\begin{array}{c}C s^{137} \\
(\mu C i) \\
(B \& W / S A I)^{e, f} \\
\end{array}$ \\
\hline 42 & 10 & 52512 & -- & -- & -- & $5.0 E-2 /$ & $3.6 E-1 /$ \\
\hline 38 & $11^{\mathrm{h}}$ & 52513 & - & - & $4.8 E-3$ & $\begin{array}{l}1.6 \mathrm{E}-21 \\
1.6 \mathrm{E}-2\end{array}$ & $\begin{array}{l}1.1 \mathrm{E}-1 / \\
1.1 \mathrm{E}-1\end{array}$ \\
\hline
\end{tabular}

a. See Figure 7 for locations at which samples were taken.

b. For specimens 1 through 8 readings are per swipe of approximately $100 \mathrm{~cm}^{2}(15.5$ in.2).

c. For specimens 9 and 10 readings are for scrape samples of approximately $100 \mathrm{~cm}^{2}$.

d. No analysis performed.

e. B\&W is Babcock and Wilcox.

f. SAI is Science Applications Incorporated.

g. The less than symbol (<) implies result below Lower Limit Detectable (LLD).

h. Specimen 11 is a paint chip from the reactor building dome. 
TABLE 9. AIRBORNE ACTIVITY, NOVEMBER 13, 1980

\begin{tabular}{|c|c|c|c|c|c|c|c|c|c|c|c|}
\hline $\begin{array}{l}\text { Data } \\
\text { Acquisition } \\
\text { Task Number } \\
\end{array}$ & Specimen ${ }^{a}$ & $\begin{array}{l}\text { Sample } \\
\text { Number }\end{array}$ & $\begin{array}{c}{[-31} \\
(u C i / m]) \\
(B \& W)^{b, c} \\
\end{array}$ & $\begin{array}{l}\text { Cs }-134 \\
(\mathrm{uCi} / \mathrm{ml}) \\
(\mathrm{B} \& \mathrm{~W})^{b} \\
\end{array}$ & $\begin{array}{c}\mathrm{Cs}-137 \\
(\mu \mathrm{C} i / \mathrm{ml}) \\
(\mathrm{B} \& \mathrm{~W})^{b} \\
\end{array}$ & $\begin{array}{c}C 0-58 \\
(\mu C i / m 1) \\
(B \& W)^{b, C} \\
\end{array}$ & $\begin{array}{l}C 0-60 \\
(\mu C i / m l) \\
(B \& W)^{b, c} \\
\end{array}$ & $\begin{array}{l}S r-90 \\
(\nu C i / m l) \\
(S A I)^{d} \\
\end{array}$ & $\begin{array}{c}\text { Gross } \\
\text { alpha } \\
(u C i / m l) \\
(B \& W)^{b, c, d} \\
\end{array}$ & $\begin{array}{c}\text { Gross } \\
\text { alpha } \\
\& \text { gamma } \\
(u C i / m l) \\
(B \& W)^{C} \\
\end{array}$ & Comments \\
\hline$N / A$ & BZA & 52320 & $<9.3 E-10$ & $2.0 E-8$ & $1.4 \mathrm{E}-7$ & $<3.7 E-10$ & $<3.9 E-10$ & $4.6 E-9$ & $--e$ & 1.17E-7 & \\
\hline$N / A$ & $B Z A$ & 52321 & $<7.1 E-10$ & $1.4 E-8$ & $1.0 \mathrm{E}-7$ & $<2.2 E-10$ & $<2.8 \mathrm{E}-10$ & $3.4 \mathrm{E}-9$ & -- & $7.69 \mathrm{E}-8$ & \\
\hline$N / A$ & $B Z A$ & 52322 & $<5.4 E-10$ & $1.7 E-8$ & $1.3 E-7$ & $<2.6 \mathrm{E}-10$ & $<1.4 E-10$ & $4.1 E-9$ & -- & $8.28 E-8$ & \\
\hline$N / A$ & $B Z A$ & 52348 & $<4.0 E-10$ & $7.7 E-9$ & $6.4 \mathrm{E}-8$ & $<1.7 E-10$ & $<1.5 \mathrm{E}-10$ & $2.5 E-9$ & -- & $6.08 \mathrm{E}-8$ & \\
\hline$N / A$ & $B Z A$ & 52349 & $<8.4 E-10$ & $1.68 \mathrm{E}-10$ & $1.1 E-8$ & $<2.5 E-10$ & $<4.2 E-10$ & $4.3 E-9$ & $2.33 E-12$ & $9.03 E-8$ & \\
\hline$N / A$ & $B Z A$ & 52350 & $<7.9 E-10$ & $1.8 E-8$ & $1.4 \mathrm{E}-7$ & $<3.8 E-10$ & $<2.6 \mathrm{E}-10$ & $4.9 E-9$ & $2.33 E-12$ & $1.14 \mathrm{E}-7$ & \\
\hline$N / A$ & BZA & 52351 & $<3.5 E-10$ & $1.2 E-8$ & $9.4 E-8$ & $<1.5 E-10$ & $<7.7 E-11$ & $6.4 E-9$ & $1.22 E-12$ & $6.96 \mathrm{E}-8$ & \\
\hline$N / A$ & $B Z A$ & 52352 & $<4.0 \mathrm{E}-10$ & 7.1E-9 & 4. $7 E-8$ & $<1.5 E-10$ & $<2.2 E-10$ & $2.5 E-9$ & $1.22 E-12$ & $4.57 E-8$ & \\
\hline N/A & $B Z A$ & 52353 & $<3.8 \mathrm{E}-10$ & $7.2 E-9$ & $5.7 E-8$ & $<1.4 \mathrm{E}-10$ & Cl. $4 \mathrm{E}-10$ & $4.4 E-9$ & $1.22 E-12$ & $5.39 E-8$ & \\
\hline$N / A$ & BZA & 52354 & $<2.6 E-10$ & $6.5 E-9$ & $5.0 E-8$ & $<1.2 E-10$ & $<7.7 \mathrm{E}-11$ & $3.1 E-9$ & $1.22 E-12$ & $5.02 E-8$ & \\
\hline$N / A$ & $B Z A$ & 52355 & $<3.7 E-10$ & $5.5 E-9$ & $3.6 \mathrm{E}-8$ & $<1.3 \mathrm{E}-10$ & $<2.2 \mathrm{E}-10$ & $2.1 E-9$ & $1.22 E-12$ & $3.26 \mathrm{E}-8$ & \\
\hline$N / A$ & $B Z A$ & 52326 & $<3.5 E-10$ & $5.5 E-9$ & $4.3 E-8$ & $<1.3 \mathrm{E}-10$ & $<1.4 E-10$ & 3. IE-9 & $1.22 \mathrm{E}-12$ & 4.35E -8 & \\
\hline$N / A$ & $\begin{array}{l}\text { High-volume } \\
\text { dir sample }\end{array}$ & 52319 & $<6.2 \mathrm{E}-11$ & $4.6 E-9$ & 3. $3 E-8$ & $<2.1 E-11$ & $<1.0 \mathrm{E}-11$ & $1.9 E-9$ & $4.52 E-12$ & -- & $\begin{array}{l}\text { Sample was taken } 18 \text { in. } \\
\text { (approximately } 47 \mathrm{~cm} \text { ) from } \\
\text { the south } 0-r i n g \text { wall and } \\
\text { approximately } 18 \mathrm{in} \text {. atove } \\
\text { the floor. }\end{array}$ \\
\hline $\begin{array}{l}\text { a. Lapel air } \\
\text { b. Rivi is Ba } \\
\text { c. The less } \\
\text { d. Sil is So } \\
\text { e. ine dash }\end{array}$ & $\begin{array}{l}\text { sample. } \\
\text { bcock and wil } \\
\text { than symbol } \\
\text { ience Applica } \\
(--) \text { indicate }\end{array}$ & $\begin{array}{l}\text { cox. } \\
\text { ) implife } \\
\text { tions Ine } \\
\text { no anal }\end{array}$ & result be & ow Lower & it Dete & ble (LLD). & & & & & \\
\hline
\end{tabular}




\section{Decontamination Test}

Smear samples 1 through 8 were taken in the center of a test area of approximately $3700 \mathrm{~cm}^{2}\left(4 \mathrm{ft}^{2}\right)$. This area is located on Elevation 305 between the \#2 PAL entry ramp and the D-ring as shown in Figure 7. Sequence of the smears was as follows:

Smears 1 and 2: prior to any decontamination operations

Smears 3 and 4: after demineralized water wash

Smears 5 and 6: after Radiac wash

Smears 7 and 8: after Radiac scrub.

Scrape sample 9 was taken outside the decontamination area. Scrape sample 10 was taken in the decontamination area after the final smears. Sample 11 is a paint chip found on the floor at Elevation 347 . The chip came from the reactor building dome and is approximately $300 \mathrm{~cm}^{2}$ (approximately 46.5 in. $^{2}$ ).

Data from the test are presented in Table 8 .

\section{Airborne Activity}

Air samples were taken during entry 4. Airborne activity data are presented in Table 9. 
Twe lve men participated in entry 4. Most noted apparent superficial damage (e.g., bowed elevator door, mud and rust stains at various locations, missing power rail for the polar crane).

Among other comments made during debriefing are the following:

- After measurement, it appears very feasible to build a new airlock inside the equipment airlock. Demineralized water connections with Hanson fittings exist at both corners of the D-ring.

- The grating by the B-core flood tank is so heavy that even two men may not be able to lift it.

- Pressure on the A steam generator registered approximately $2.5 \mathrm{lb}$ on the local gauge, the same reading as is indicated in the M20 area.

- Radiation levels were higher on the B steam generator side than on the A steam generator side.

- Gamma radiation appears to be coming through the floor of Elevation 305. During the decontamination test, the gamma radiation dropped significantly after the initial wash, but the Radiac wash and scrub brought no further reduction.

- Lighting levels inside the reactor building are inadequate for high-quality video. The portable flood lights used were difficult to maneuver and caused reflections. Some locations could not be illuminated at all, and the cameras could not gain access to some areas. 


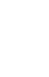


$+$.

, 


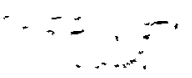$$
\text { E. }
$$

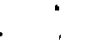

